\title{
A Low-Power Scheme for Localization in Wireless Sensor Networks
}

\author{
Jorge Juan Robles, Sebastian Tromer, Monica Quiroga, and Ralf Lehnert \\ Dresden University of Technology, Dresden, 01069 Germany \\ \{robles, lehnert\} @ifn.et.tu-dresden.de
}

\begin{abstract}
One of the most challenging issues in the design of system for Wireless Sensor Networks (WSN) is to keep the energy consumption of the sensor nodes as low as possible. Many localization systems require that the nodes keep the transceiver active during a long time consuming energy. In this work we propose a scheme to reduce the energy consumption of the mobile nodes that need to know their positions. Our strategy consists of decreasing the idle listening and an optimized allocation of the localization tasks on the nodes. Thus, the nodes that are externally powered calculate the position for the resourceconstrained nodes. The scheme is based on a low-power IEEE 802.15.4 nonbeacon enabled network.
\end{abstract}

Keywords: localization scheme, positioning algorithm, idle listening, wireless sensor networks.

\section{Introduction}

In a WSN the sensor node's position is used, e.g. in geographical routing, clustering techniques and context-based applications.

For the position estimation many localization algorithms utilize the information of the distance between nodes and the position of certain nodes, called anchors. The distance between two nodes can be calculated by measuring the received signal strength (RSS) at the receiver and considering an appropriate attenuation model. Unfortunately the RSS measurements have a big dispersion due to environment characteristics. Therefore many RSS measurements are required to achieve a good accuracy at expense of additional energy consumption. In our scenario the anchors are fixed and externally powered whereas the mobile nodes are battery-operated. The mobile nodes need to know their positions.

The goal of the proposed scheme is to provide, in an efficient way, the necessary information to the localization algorithm for the position calculation. Thus, RSSbased localization algorithms, such as Multilateration and Centroid [2] could be independently implemented on our scheme.

\section{Idle Listening in the Localization Algorithms}

Many localization systems, like in [3], are similar to the following simplified protocol: 
A) The Mobile node (MN) broadcasts a localization request.

B) The anchors, which receive the request, send to the MN a certain number of packets containing relevant information for the position calculation.

C) The MN measures the RSS of each packet. Using these measurements and the received information, the $\mathrm{MN}$ executes a localization algorithm. It can also send the information to an anchor or a central unit.

Considering an IEEE 802.15.4 beaconless network [1], in the described basic protocol, the MN has to be active for a long time to receive enough packets from the anchors. This is due to the time that is required by the anchor for a successful transmission (CSMA/CA is used by the MAC layer). During the time between successful transmissions of the anchors the $\mathrm{MN}$ is listening (because it does not know when the next packet arrives) consuming a high amount of energy. This is called idle listening.

\section{Low-Power Scheme for Localization}

The low-power scheme tries to reduce the energy consumption of the MNs by reducing the idle listening period and incrementing the sleep periods. The network maintains a global synchronization. The anchors are active all the time and have the information related to the synchronization. The proposed scheme divides the localization task into four different phases (Fig. 1):

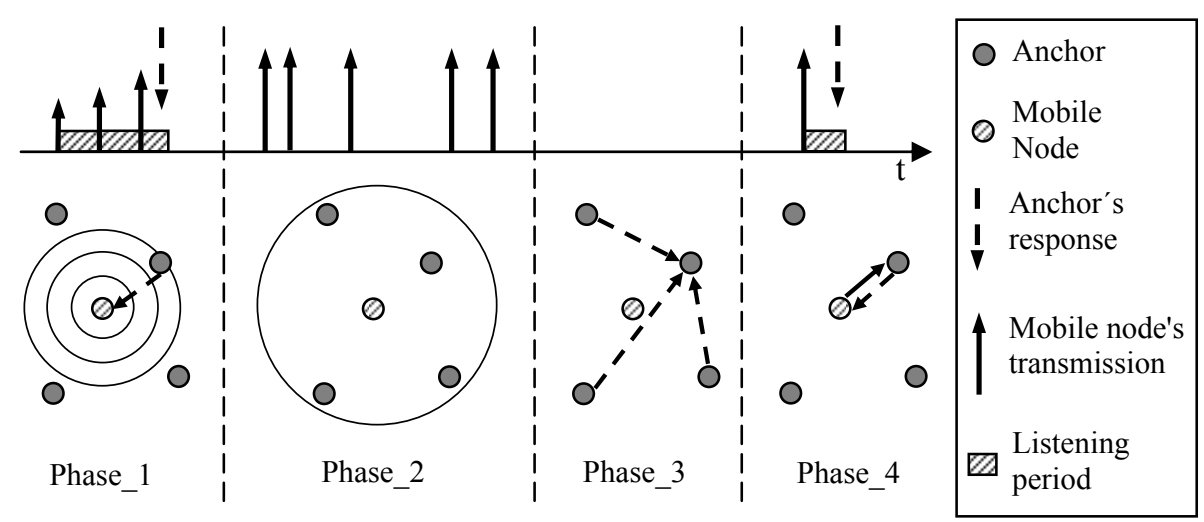

Fig. 1. Operation of the low-power scheme for a MN over the time

1) Phase_1: The MN broadcasts a localization request at minimal power transmission. If the anchors receive the request, they answer indicating the duration of the phases. If the $\mathrm{MN}$ does not receive any answer it tries to send another request at a higher transmission power. This process is to ensure that the nearest anchors to the MN can answer first. When the mobile node receives the first answer, it goes into sleep mode and waits for the following phase. 
2) Phase_2: The MN broadcasts a certain number of packets at maximal power transmission. This process is random over the time decreasing the collision probability with other MNs. During the time between transmissions the MN goes into sleep mode in order to save energy. The anchors, which receive the packets, take the corresponding RSS measurements. The packets contain the information of the "selected anchor", which is the anchor that answered in the phase_1.

3) Phase_3: During all the phase_3 the mobile node is in sleep mode. The selected anchor is in charge of the position estimation of the MN. Thus, the anchors that have received packets in the phase_2, send their positions and RSS measurements to the selected anchor. The selected anchor executes a localization algorithm in order to estimate the MN position.

4) Phase_4: If the MN needs to know its position, it sends an information request to the selected anchor. The answer of the selected anchor contains the calculated position. After this task the MN goes into sleep mode.

If the anchors receive a localization request outside of phase_1 then they send a packet to the $\mathrm{MN}$ indicating when the phase_1starts.

The energy consumption of an IEEE 802.15.4 transceiver was studied for the basic protocol described in the section two and the low-power scheme (see Fig. 2). In our first analysis the following assumptions were taken:

1) The scenario consists of four anchors and one mobile node.

2) CSMA/CA needs $5 \mathrm{~ms}$ for a successful transmission [5].

3) The duration of each transmission is $1,5 \mathrm{~ms}$.

4) The information about the energy consumption in the different modes was obtained from the specifications of the transceiver AT86RF230 [4].

5) In the basic protocol each anchors transmits 5 packets to the $\mathrm{MN}$.

6) In the basic protocol the MN listens during $150 \mathrm{~ms}$ and sleeps $850 \mathrm{~ms}$.

7) In the phase_1 the MN transmits 2 times waiting $10 \mathrm{~ms}$ in each trial.

8) In the phase_2 the MN sends 5 packets.

9) In the phase_4 the MN receives a response after 10ms.

10) The duration of the localization period is $1000 \mathrm{~ms}$.

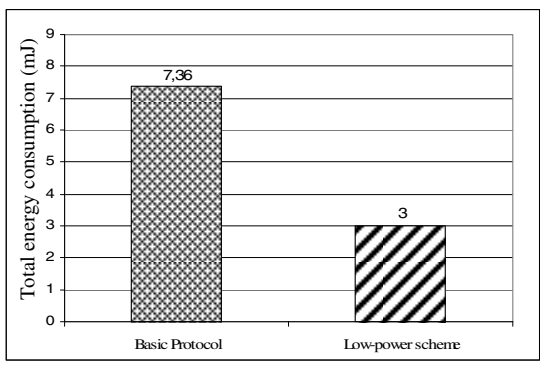

(a)

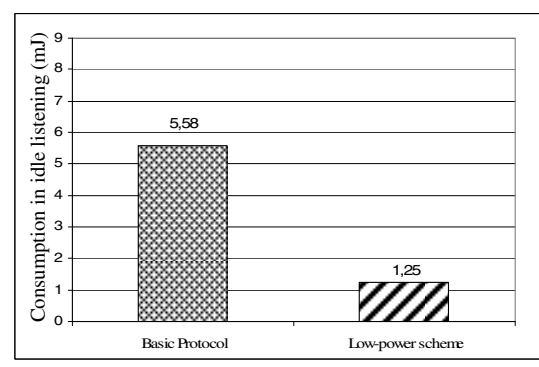

(b)

Fig. 2. a) Total energy consumption of the MN's transceiver in one localization period. b) Energy consumption of the MN's transceiver due to idle listening in one localization period. 


\section{Conclusions}

We present the main operation of a novel low-power scheme for localization algorithms. It can reduce the energy consumption of the mobile nodes by decreasing the idle listening and increasing the sleep period. The future work will be focused on an extended performance evaluation of the proposed scheme.

\section{References}

1. IEEE standard 802.15.4-2006 (2006), http: / / standards.ieee.org/getieee802/802.15.html

2. Reichenbach, F., Blumenthal, J., Timmermann, D.: Comparing the Efficiency of Localization Algorithms with the Power-Error-Product (PEP). In: 28th International conference on Distributed Computing Systems Workshops, Beijing, China (2008)

3. Lorincz, K., Welsh, M.: MoteTrack: a robust, decentralized approach to RF-based location tracking. Personal and Ubiquitous Computing 11(6) (2007)

4. Datasheet IEEE 802.15.4 transceiver AT86RF240, http://www.atmel.com

5. Rohm, D., Goyal, M., Hosseini, H., Divjak, A., Bashir, Y.: Configuring Beaconless IEEE 802.15.4 Networks Under Different Traffic Loads. In: International Conference on Advanced Information Networking and Applications, Bradford, UK (2009) 